\title{
Mathematical Modeling of Finite Quantum Systems
}

\author{
Vladimir V. Kornyak \\ Laboratory of Information Technologies \\ Joint Institute for Nuclear Research \\ 141980 Dubna, Russia \\ kornyak@jinr.ru
}

\begin{abstract}
We consider the problem of quantum behavior in the finite background. Introduction of continuum or other infinities into physics leads only to technical complications without any need for them in description of empirical observations. The finite approach makes the problem constructive and more tractable. We argue that quantum behavior is a natural consequence of symmetries of dynamical systems. It is a result of fundamental impossibility to trace identity of indistinguishable objects in their evolution - only information about invariant combinations of such objects is available. We demonstrate that any quantum dynamics can be embedded into a simple permutation dynamics. Quantum phenomena, such as interferences, arise in invariant subspaces of permutation representations of the symmetry group of a system. Observable quantities can be expressed in terms of the permutation invariants.
\end{abstract}

\section{Introduction}

Unitary operators in Hilbert spaces - in fact, unitary representations of some symmetry groups - lie in the core of mathematical descriptions of quantum phenomena. We can assume finiteness of these groups without any risk to destroy the physical content of the problem, since metaphysical choice between "finite" and "infinite" can not lead to any empirically observable consequences. Moreover, there are strong experimental evidences that finite groups of relatively small orders underlie some fundamental physical processes [13/7/11|5|6|1|15|2]. These evidences come mainly from the flavor physics, especially in the lepton sector. The origin of these small groups is unclear in the context of currently accepted theories like, e.g., the Standard Model.

Let us recall some standard facts from the group theory [4]:

- Any linear representation of a finite group $G=\left\{g_{1}, \ldots, g_{M}\right\}$ is unitary.

- Any irreducible representation is contained in the regular representation matrix version of permutation action of the group $\mathrm{G}$ on its elements.

- Any set $\Omega=\left\{\omega_{1}, \ldots, \omega_{N}\right\}$ on which $G$ acts transitively by permutations is equivalent to a set of cosets of some subgroup in $\mathrm{G}$. 
Combination of these facts leads to the conclusion that any quantum problem with evolution operators $\mathrm{U}\left(\mathrm{g}_{i}\right)$ belonging to a representation $\mathrm{U}$ of $\mathrm{G}$ in $\mathrm{K}$ dimensional Hilbert space $\mathcal{H}_{\mathrm{K}}$ can be reduced to permutations of $\mathrm{N} \geq \mathrm{K}$ things. If $\mathrm{N}>\mathrm{K}$ then the permutation representation $\mathrm{P}$ in $\mathrm{N}$-dimensional Hilbert space $\mathcal{H}_{\mathrm{N}}$ has the structure $\mathrm{P} \cong \mathbf{1} \oplus \mathrm{U} \oplus \mathrm{V}$. Additional "hidden parameters" appearing due to increase of Hilbert space dimension in no way can effect on the data relating to the space $\mathcal{H}_{\mathrm{K}}$ since both this space and its complement are invariant subspaces in $\mathcal{H}_{\mathrm{N}}$. Quantum phenomena, such as, e.g., destructive interference, can be observed in proper invariant subspaces of the representation P. More detailed examination - starting with the fact that all eigenvalues of group representations are roots of unity - leads to the conclusion that in "finite" quantum formalism the field of complex numbers should be replaced by some extension $\mathcal{F}$ of rational numbers with abelian Galois group. This extension depends on the structure of underlying group $\mathrm{G}$. The field $\mathcal{F}$ is a subfield of some cyclotomic field (the Kronecker-Weber theorem). Hermitian operators describing observables in quantum formalism are elements of representation of group algebra over $\mathcal{F}$. All ingredients of quantum theory - like, e.g., the Heisenberg uncertainty principle - are obtained in the standard way. Observable data in the space $\mathcal{H}_{\mathrm{K}}$ can be expressed in terms of permutation invariants relating to the space $\mathcal{H}_{\mathrm{N}}$.

The permutation representation $\mathrm{P}$ makes sense in the $\mathrm{N}$-dimensional module $\mathrm{H}_{\mathrm{N}}$ over the semi-ring $\mathbb{N}=\{0,1,2, \ldots\}$ of natural numbers. Introduction of $\mathbf{H}_{\mathrm{N}}$ is equivalent to prescribing natural weights ("multiplicities of occurrences" or "population numbers") to the elements of underlying set $\Omega$. The Hilbert space $\mathcal{H}_{\mathrm{N}}$ is derived from $\mathrm{H}_{\mathrm{N}}$ by extending the semi-ring $\mathbb{N}$ to the field $\mathcal{F}$. If quantum amplitudes in $\mathcal{H}_{\mathrm{K}}$ are obtained as projections of "natural" vectors from $\mathrm{H}_{\mathrm{N}} \subset$ $\mathcal{H}_{\mathrm{N}}$, then the Born probabilities in $\mathcal{H}_{\mathrm{K}}$ appear to be rational numbers. This is compatible with the frequency interpretation of probability for finite sets: the probability is the ratio of singled out combinations to the total number of combinations under consideration. The interpretational issues like "wavefunction collapse", "many-worlds", "many-minds" etc. lose any meaning in the finite background. The quantum phenomena arise as manifestation of fundamental impossibility to trace identity of indistinguishable objects in their evolution only information about invariant combinations of such objects is available.

\section{Basic Structures}

We consider evolution of dynamical systems in the discrete time $\mathcal{T}$. We will assume that $\mathcal{T}=\mathbb{Z}$ or $\mathcal{T}=[0,1, \ldots, T]$ for some $T \in \mathbb{N}$.

Classical states of dynamical system form a finite set $\Omega=\left\{\omega_{1}, \ldots, \omega_{N}\right\}$ of some entities. Classical evolution or trajectory of the dynamical system is a sequence $\ldots, \omega_{t-1}, \omega_{t}, \omega_{t+1}, \ldots \in \Omega^{\mathcal{T}}$. We assume the existence of a symmetry group $\mathrm{G}=\left\{\mathrm{g}_{1}, \ldots, \mathrm{g}_{\mathrm{M}}\right\} \leq \operatorname{Sym}(\Omega)$ acting on the entities $\Omega$.

We will argue that quantum evolution can be defined as a sequence of permutations $\ldots p_{t-1}, p_{t}, p_{t+1} \ldots$, where $p_{t}=\Omega a_{t}$ is the permutation of the set $\Omega$ by a group element $a_{t} \in \mathrm{G}$. A natural condition here is that at any moment 
$t$ only information on invariant combinations of elements from $\Omega$ is available, whereas the permutation $p_{t}$ itself is unobservable. More precisely, the information about arrangement of elements from $\Omega$ corresponding to $p_{t}$ does not make sense without a "reference frame" (or "observer").

In physics the set $\Omega$ usually has a special structure of a set of functions

$$
\Omega=\Sigma^{\mathrm{X}}
$$

on a space $\mathbf{X}=\left\{\mathrm{x}_{1}, \ldots, \mathrm{x}_{|\mathrm{X}|}\right\}$ with values in a set $\Sigma=\left\{\sigma_{1}, \ldots, \sigma_{|\Sigma|}\right\}$ of local states.

We assume that both the space $\mathbf{X}$ and the local states $\Sigma$ possess nontrivial groups of space $\mathrm{F}=\left\{\mathrm{f}_{1}, \ldots, \mathrm{f}_{|\mathrm{F}|}\right\} \leq \operatorname{Sym}(\mathrm{X})$ and internal $\Gamma=\left\{\gamma_{1}, \ldots, \gamma_{|\Gamma|}\right\} \leq$ $\operatorname{Sym}(\Sigma)$ symmetries, respectively. In principle, the most general symmetry group $\mathrm{G}$ for the set of states having form (11) can be computed with the help of modern algorithm: 1. Of course, this might be combinatorially difficult task. However, we can easily construct some group $\mathrm{G}$ directly from the groups $\mathrm{F}$ and $\Gamma$. The construction - generalizing what is used in physical theories - is the following equivalence class of split extensions

$$
\mathbf{1} \rightarrow \Gamma^{\mathrm{X}} \rightarrow \mathrm{G} \rightarrow \mathrm{F} \rightarrow \mathbf{1}
$$

where $\Gamma^{\mathrm{X}}$ is the group of $\Gamma$-valued functions on the space $\mathrm{X}$. Any equivalence class is determined by an antihomomorphism $\mu: \mathrm{F} \rightarrow \mathrm{F}$. The term 'antihomomorphism' means that $\mu(a b)=\mu(b) \mu(a)$ for $a, b \in \mathrm{F}$. For example, choosing the trivial $\mu(a)=1$ and the natural $\mu(a)=a^{-1}$ antihomomorphisms we obtain, respectively:

- the direct product $\mathrm{G}=\Gamma^{\mathrm{X}} \times \mathrm{F}$ (this is the standard choice in physical theories)

- the wreath product $\mathrm{G}=\Gamma \mathrm{\lambda}_{\mathrm{X}} \mathrm{F} \equiv \Gamma^{\mathrm{X}} \rtimes \mathrm{F}$ ( $\rtimes$ stands for the semidirect product).

In 10. we gave explicit formulas for group operations in $G$ defined by (2) in terms of operations in $\mathrm{F}$ and $\Gamma$.

An important property of dynamical systems with spaceis the presence of non-trivial gauge connections. The gauge structures lead to observable physical consequences: the curvatures of non-trivial connections describe forces in physical theories. Another important topic involving the space structure is the spin/statistics relation. On the other hand there are many problems, for example, quantum computing, where any underlying space is inessential.

\section{$3 \quad$ Finite Quantum Models}

As is well known, all approaches to quantization are equivalent to the traditional matrix formulation of quantum mechanics where the evolution of a system from an initial to a final state is described by an evolution matrix $U$ :

\footnotetext{
${ }^{1}$ The algorithm designed by B. McKay [12] is considered as the most efficient to date. Its main target are graphs, but its universal scheme can be adjusted without excessive efforts for computing symmetries of other combinatorial structures.
} 
$\left|\psi_{0}\right\rangle \rightarrow\left|\psi_{T}\right\rangle=U\left|\psi_{0}\right\rangle$. The evolution matrix of a quantum dynamical system can be represented as the product of matrices corresponding to elementary time steps: $U=U_{T \leftarrow T-1} \cdots U_{t \leftarrow t-1} \cdots U_{1 \leftarrow 0}$. We will follow the evolution matrix approach throughout this paper.

The main ingredients of the standard quantum mechanics are the following:

1. Quantum description deals with unitary operators $U$ acting in a Hilbert space $\mathcal{H}$ over the field of complex numbers $\mathbb{C}$. The elements $|\psi\rangle \in \mathcal{H}$ of the space are called "states", "state vectors", "wave functions", "amplitudes" etc. The operators $U$ belong to the general unitary group $\operatorname{Aut}(\mathcal{H})$ acting in $\mathcal{H}$.

2. Quantum mechanical particles are associated with unitary representations in $\mathcal{H}$ of some symmetry groups. The representations are called "singlets", "doublets", "triplets" etc. in accordance with their dimensions. The multidimensional representations describe spin

3. Quantum mechanical evolution is an unitary transformation of the initial state vector $\left|\psi_{\text {in }}\right\rangle$ into the final $\left|\psi_{\text {out }}\right\rangle=U\left|\psi_{\text {in }}\right\rangle$. In the continuous time, an elementary step of evolution is described by the Schrödinger equation

$$
i \frac{\mathrm{d}}{\mathrm{d} t}|\psi\rangle=H|\psi\rangle
$$

where $H$ is a Hermitian operator called energy operator or Hamiltonian.

4. Quantum mechanical experiment (observation, "measurement") is a comparison of the state $|\psi\rangle$ of a system with the state $|\phi\rangle$ of an apparatus.

5. In accordance with the Born rule, the probability $\mathbf{P}(\phi, \psi)$ to register a particle described by $|\psi\rangle$ by apparatus tuned to $|\phi\rangle$ is equal to $\frac{|\langle\phi \mid \psi\rangle|^{2}}{\langle\phi \mid \phi\rangle\langle\psi \mid \psi\rangle}$.

6. Quantum observables are described by Hermitian operators acting in the Hilbert space $\mathcal{H}$.

Our aim is to reproduce all this in the constructive finite background. Our strategy - in accordance with the Occam principle - will be to avoid introduction of entities unless we really need them. Keeping these lines we come to the following:

$1^{\prime}$. The Hilbert space $\mathcal{H}$ over the field $\mathbb{C}$ should be replaced by a K-dimensional Hilbert space $\mathcal{H}_{\mathrm{K}}$ over an abelian number field $\mathcal{F}$ - an extension of the rationals $\mathbb{Q}$ with an abelian Galois group [14. The unitary operators $U$ belong now to an unitary representation $\mathrm{U}$ of a finite group $\mathrm{G}=\left\{\mathrm{g}_{1}, \ldots, \mathrm{g}_{\mathrm{M}}\right\}$ in the space $\mathcal{H}_{\mathrm{K}}$. The field $\mathcal{F}$ is determined by the structure of the group $\mathrm{G}$ and its representation $\mathrm{U}$.

$2^{\prime}$. The notion of quantum particle remains the same as in the standard quantum mechanics.

$3^{\prime}$. It is clear that now we have only finite number of possible evolutions:

$$
U_{j} \in\left\{\mathrm{U}\left(\mathrm{g}_{1}\right), \ldots, \mathrm{U}\left(\mathrm{g}_{j}\right), \ldots, \mathrm{U}\left(\mathrm{g}_{\mathrm{M}}\right)\right\} .
$$

Obviously we do not need any analog of the Schrödinger equation at all. Though formally one can always introduce Hamiltonians by the formula 
$H_{j}=i \ln U_{j} \equiv \sum_{k=0}^{p-1} \lambda_{k} U_{j}^{k}$, where $p$ is period of $U_{j}$ (i.e., minimal $p>0$ such that $\left.U_{j}^{p}=\mathrm{I}\right), \lambda_{k}$ 's are some coefficient. 2 .

$4^{\prime}$. The notion of observation remains without changes.

$5^{\prime}$. The formula for Born's probability remains the same

$$
\mathbf{P}(\phi, \psi)=\frac{|\langle\phi \mid \psi\rangle|^{2}}{\langle\phi \mid \phi\rangle\langle\psi \mid \psi\rangle}
$$

But some conceptual refinement is needed. In the finite background the only reasonable interpretation of probability is the frequency interpretation: the probability is the ratio of the number of singled out combinations to the total number of combinations under consideration. So we expect that if all things are arranged correctly, then formula (3) must give rational numbers. We will use this as a guiding principle.

$6^{\prime}$. Hermitian operators describing observables in quantum formalism can be expressed in terms of the group algebra representation:

$$
A=\sum_{k=1}^{\mathrm{M}} \alpha_{k} \mathrm{U}\left(\mathrm{g}_{k}\right) .
$$

Of course, to provide hermiticity appropriate conditions should be imposed on the coefficients $\alpha_{k}$.

Note that other elements of the quantum theory are obtained in the finite background in the standard way. For example, as is well known, the Heisenberg uncertainty principle follows from the Cauchy-Bunyakovsky-Schwarz inequality

$$
\langle A \psi \mid A \psi\rangle\langle B \psi \mid B \psi\rangle \geq|\langle A \psi \mid B \psi\rangle|^{2} .
$$

In fact, in our consideration we deal with the Cauchy inequality - two others extended (41) to the continuous case. Obviously, the Cauchy inequality is equivalent to the standard property of any probability $\mathbf{P}(A \psi, B \psi) \leq 1$.

\section{Permutations, Representations and Numbers}

All transitive actions of a finite group $G=\left\{g_{1}, \ldots, g_{M}\right\}$ on finite sets can easily be described [4. Any such set $\Omega=\left\{\omega_{1}, \ldots, \omega_{\mathrm{N}}\right\}$ is in one-to-one correspondence with a set of right $H \backslash \mathrm{G}$ (or left $\mathrm{G} / H$ ) cosets of some subgroup $H \leq \mathrm{G}$. The set $\Omega$ is called a homogeneous space of the group G (G-space for short). Action of G

\footnotetext{
${ }^{2}$ These coefficients contain the non-algebraic element $\pi$ which is an infinite sum of elements from $\mathcal{F}$. In other words, the $\lambda_{k}$ 's are elements of a transcendental extension of $\mathcal{F}$ - the logarithmic function is essentially a construction from the continuous mathematics dealing with infinities.
} 
on $\Omega$ is faithful, if the subgroup $H$ does not contain normal subgroups of G. We can write the action in the form of permutations

$$
\pi(g)=\left(\begin{array}{c}
\omega_{i} \\
\omega_{i} g
\end{array}\right) \sim\left(\begin{array}{c}
H a \\
H a g
\end{array}\right), \quad g, a \in \mathrm{G}, \quad i=1, \ldots, \mathrm{N},
$$

or, equivalently, in the form of matrix with entries 0 and 1

$$
\pi(g) \rightarrow \mathrm{P}(g)=\left(\mathrm{P}(g)_{i j}\right), \quad \text { where } \mathrm{P}(g)_{i j}=\delta_{\omega_{i} g, \omega_{j}} ; i, j=1, \ldots, \mathrm{N} .
$$

Here $\delta_{\alpha, \beta}$ is the Kronecker delta on $\Omega$. Mapping (6) is called the permutation representation.

Maximal transitive set $\Omega$ is the set of all elements of the group $\mathrm{G}$ itself, i.e., the set of cosets of the trivial subgroup $H=\{\mathbf{1}\}$. The corresponding action and matrix representation are called regular. One of the central theorems in the representation theory states that any irreducible representation of a finite group is contained in the regular representation.

Representation (6) makes sense over any number system with 0 and 1 . A very natural number system is the semi-ring of natural numbers $\mathbb{N}=\{0,1,2, \ldots\}$. With this semi-ring we can attach counters to elements of the set $\Omega$. These counters (natural numbers) can be interpreted as "multiplicities of occurrences" or "population numbers" of elements $\omega_{i}$ in the state of a system involving elements from $\Omega$. Such state can be represented by the vector with natural components

$$
|n\rangle=\left(\begin{array}{c}
n_{1} \\
\vdots \\
n_{\mathrm{N}}
\end{array}\right) .
$$

Thus, we come to the representation of the group $\mathrm{G}$ in an $\mathrm{N}$-dimensional module $\mathrm{H}_{\mathrm{N}}$ over the semi-ring $\mathbb{N}$. Representation (6) when applied to vector (7) simply permutes its components. For further development we can turn the module $\mathrm{H}_{\mathrm{N}}$ into an $\mathrm{N}$-dimensional Hilbert space $\mathcal{H}_{\mathrm{N}}$ by extending $\mathbb{N}$ to some field.

The main field in the theory of representations (and hence in the quantum mechanics) is the field of complex numbers $\mathbb{C}$. The reason for this choice is simple: the field $\mathbb{C}$ is algebraically closed, so no complications can be expected in solving characteristic equations and, hence, in the whole linear algebra. However, the field $\mathbb{C}$ is excessively large - most of its elements are non-constructive. So let us consider the problem more carefully.

First of all, we do not need to solve arbitrary characteristic equations: any representation is subrepresentation of some permutation representation, and eigenvalues of any permutation representation are roots of unity. This is clear from the easily calculated characteristic polynomial of permutation matrix (6)

$$
\chi_{\mathrm{P}(g)}(\lambda)=\operatorname{det}(\mathrm{P}(g)-\lambda \mathrm{I})=(\lambda-1)^{k_{1}}\left(\lambda^{2}-1\right)^{k_{2}} \cdots\left(\lambda^{n}-1\right)^{k_{n}},
$$

where $k_{i}$ is the number of cycles of the length $i$ in permutation (5). To provide unitarity of representations we use square roots of their dimensions as normalizing coefficients. In fact, all irrationalities (square roots of natural numbers and 
roots of unity) are elements of the same nature - they are cyclotomic integers, i.e., combinations of roots of unity with integer coefficients (that can be made natural by using appropriate identities for roots of unity).

Thus, the basic elements of the number system, we are going to construct, are natural numbers and linear combinations (with natural coefficients) of roots of unity of some degree $\mathcal{P}$ depending on the structure of the group $\mathrm{G}$. This degree is called conductor. Starting with these basic elements, via standard mathematical derivation, we come to a minimal abelian number field $\mathcal{F}$ containing these basics. In particular, such field can be computed with the help of the computer algebra system GAP 3 . The command Field (gens) of this system returns the smallest field that contains all elements from the list of irrationalities gens. The field $\mathcal{F}$ can be embedded into the field $\mathbb{C}$, but we do not need this possibility. Purely algebraic properties of $\mathcal{F}$ are sufficient for all manipulations in the Hilbert space $\mathcal{H}_{\mathrm{N}}$ and its subspaces.

All irrationalities are intermediate elements of quantum description that disappear in the final expressions for quantum observables - this is a refinement of the usual relationship between the complex and real numbers in the standard quantum mechanics: the intermediate values may be complex whereas the final observables are to be real.

\section{$5 \quad$ Embedding Quantum System into Permutations}

It follows from the above that any K-dimensional representation $\mathrm{U}$ can be extended to an $\mathrm{N}$-dimensional representation $\widetilde{\mathrm{U}}$ in a Hilbert space $\mathcal{H}_{\mathrm{N}}$, in such a way that the representation $\widetilde{U}$ corresponds to the permutation action of the group $\mathrm{G}$ on some $\mathrm{N}$-element set of entities $\Omega=\left\{\omega_{1}, \ldots, \omega_{\mathrm{N}}\right\}$. This means that

$\mathrm{T}^{-1} \mathrm{PT}=\widetilde{\mathrm{U}}$, where $\mathrm{P}$ is permutation representation (6) and $\mathrm{T}$ is a transformation matrix. It is clear that $\mathrm{N} \geq \mathrm{K}$.

The case when $\mathrm{N}$ is strictly greater than $\mathrm{K}$ is most interesting. In this case the representation has the following structure

$$
\mathrm{T}^{-1} \mathrm{PT}=\left(\begin{array}{ccc}
\mathbf{1} & & \\
& \mathrm{U} & \\
& & \mathrm{V}
\end{array}\right) \equiv \mathbf{1} \oplus \mathrm{U} \oplus \mathrm{V} .
$$

Here $\mathbf{1}$ is the trivial one-dimensional representation - an obligatory component of any permutation representation. The component $\mathrm{V}$ may be empty.

Clearly, the additional "hidden parameters" - appearing due to increase of the number of dimension of space in the case $\mathrm{N}>\mathrm{K}$ - in no way can effect on the data relating to the space $\mathcal{H}_{\mathrm{K}}$ since both $\mathcal{H}_{\mathrm{K}}$ and its complement in $\mathcal{H}_{\mathrm{N}}$ are invariant subspaces of the extended space $\mathcal{H}_{\mathrm{N}}$. Thus, any quantum problem in $\mathrm{K}$-dimensional Hilbert space can be reformulated in terms of permutations of $\mathrm{N}$ things.

With the trivial assumption that the components of state vectors are arbitrary elements of the underlying field $\mathcal{F}$, we can set arbitrary (e.g., zero) data in the subspace $\mathcal{H}_{\mathrm{N}-\mathrm{K}}$ complementary to $\mathcal{H}_{\mathrm{K}}$. In this case we come - up to 
the physically inessential difference between "finite" and "infinite" — to the standard quantum mechanics reformulated in the terms of permutations.

We can drop this assumption and give more natural meaning to quantum amplitudes. Let us represent the (quantum) states of the system and apparatus in the permutation representation by the natural vectors

$$
|n\rangle=\left(\begin{array}{c}
n_{1} \\
\vdots \\
n_{\mathrm{N}}
\end{array}\right) \text { and }|m\rangle=\left(\begin{array}{c}
m_{1} \\
\vdots \\
m_{\mathrm{N}}
\end{array}\right)
$$

respectively. In accordance with the Born rule the probability to fix the system state $|n\rangle$ by apparatus tuned to $|m\rangle$ is

$$
\mathbf{P}(m, n)=\frac{\left(\sum_{i} m_{i} n_{i}\right)^{2}}{\sum_{i} m_{i}{ }^{2} \sum_{i} n_{i}{ }^{2}} .
$$

It is clear that for non-vanishing natural vectors $|n\rangle$ and $|m\rangle$ expression (8) is a rational number strictly greater than zero. This means, in particular, that it is impossible to observe destructive quantum interference here. However, the destructive interference of the vectors with natural components can be observed in the proper invariant subspaces of the permutation representation. We will demonstrate this by a simple example.

\section{Illustrative Example: Group $\mathrm{S}_{3}$}

$\mathrm{S}_{3}$ is the smallest non-commutative group. Nevertheless, $\mathrm{S}_{3}$ has important applications in physics. In particular, it describes the so-called tribimaximal mixing in the neutrino oscillations 56 . The group consists of six elements having the following representation by permutations

$$
\mathrm{g}_{1}=(), \mathrm{g}_{2}=(2,3), \mathrm{g}_{3}=(1,3), \mathrm{g}_{4}=(1,2), \mathrm{g}_{5}=(1,2,3), \mathrm{g}_{6}=(1,3,2)
$$

The group can be generated by many pairs of its elements. Let us choose, for instance, $\mathrm{g}_{2}$ and $\mathrm{g}_{6}$ as generators. $\mathrm{S}_{3}$ decomposes into the three conjugacy classes

$$
K_{1}=\left\{\mathrm{g}_{1}\right\}, K_{2}=\left\{\mathrm{g}_{2}, \mathrm{~g}_{3}, \mathrm{~g}_{4}\right\}, K_{3}=\left\{\mathrm{g}_{5}, \mathrm{~g}_{6}\right\} .
$$

The group $S_{3}$ has the following character table (a compact form to register all irreducible representations)

\begin{tabular}{l|rrr} 
& $K_{1}$ & $K_{2}$ & $K_{3}$ \\
\hline$\chi_{1}$ & 1 & 1 & 1 \\
$\chi_{2}$ & 1 & -1 & 1 \\
$\chi_{3}$ & 2 & 0 & -1
\end{tabular}.

In accordance with the physical tradition, we will denote irreducible representations by their dimensions in bold. Thus, we have here three irreducible representations $\mathbf{1}, \mathbf{1}^{\prime}$ and $\mathbf{2}$ (the last is the only faithful). For permutation representations 
playing an important role in this paper we will use analogous notation with additional overline.

Matrices of permutation representation of generators are

$$
P_{2}=\left(\begin{array}{ccc}
1 & \cdot & \cdot \\
\cdot & \cdot & 1 \\
\cdot & 1 & \cdot
\end{array}\right) \text { and } P_{6}=\left(\begin{array}{ccc}
\cdot & \cdot & 1 \\
1 & \cdot & \cdot \\
\cdot & 1 & \cdot
\end{array}\right)
$$

The eigenvalues of $P_{2}$ and $P_{6}$ are $(1,1,-1)$ and $\left(1, r, r^{2}\right)$, respectively; $r$ is a primitive $3^{d}$ root of unity satisfying to the cyclotomic polynomial $\Phi_{3}(\mathrm{r})=1+$ $r+r^{2}$.

Since (as is was mentioned above) any permutation representation contains one-dimensional invariant subspace with the basis vector $(1, \ldots, 1)^{\mathrm{T}}$, the only possible structure of decomposition of permutation representation into irreducible parts is $\overline{\mathbf{3}} \cong \mathbf{1} \oplus \mathbf{2}$ or in the explicit matrix form

$$
\widetilde{U}_{j}=\left(\begin{array}{cc}
\mathbf{1} & 0 \\
0 & U_{j}
\end{array}\right), \quad j=1, \ldots, 6,
$$

where the matrices $U_{j}$ are elements of the faithful representation 2 .

To construct decomposition (9) we should determine matrices $U_{j}$ and $\mathrm{T}$ such that $\widetilde{U}_{j}=\mathrm{T}^{-1} P_{j} \mathrm{~T}$. Additionally we impose unitarity on all the matrices. Clearly, it suffices to perform the procedure only for matrices of generators. There are different ways to construct decomposition (9). If we start with the diagonalization of $P_{6}$, we come to the following

$$
\begin{aligned}
& U_{1}=\left(\begin{array}{ll}
1 & 0 \\
0 & 1
\end{array}\right), U_{2}=\left(\begin{array}{ll}
0 & r^{2} \\
r & 0
\end{array}\right), U_{3}=\left(\begin{array}{cc}
0 & r \\
r^{2} & 0
\end{array}\right), \\
& U_{4}=\left(\begin{array}{ll}
0 & 1 \\
1 & 0
\end{array}\right), U_{5}=\left(\begin{array}{ll}
r^{2} & 0 \\
0 & r
\end{array}\right), U_{6}=\left(\begin{array}{cc}
r & 0 \\
0 & r^{2}
\end{array}\right) .
\end{aligned}
$$

The transformation matrix (up to inessential degrees of freedom for its entries) takes the following form

$$
\mathrm{T}=\frac{1}{\sqrt{3}}\left(\begin{array}{ccc}
1 & 1 & r^{2} \\
1 & r^{2} & 1 \\
1 & r & r
\end{array}\right), \quad \mathrm{T}^{-1}=\frac{1}{\sqrt{3}}\left(\begin{array}{lll}
1 & 1 & 1 \\
1 & r & r^{2} \\
r & 1 & r^{2}
\end{array}\right)
$$

The information about "quantum behavior" is encoded, in fact, in transformation matrices like (10).

$$
\text { Let }|n\rangle=\left(\begin{array}{l}
n_{1} \\
n_{2} \\
n_{3}
\end{array}\right) \text { and }|m\rangle=\left(\begin{array}{l}
m_{1} \\
m_{2} \\
m_{3}
\end{array}\right) \text { be system and apparatus state vectors in }
$$

the "permutation" basis. Transformation of these vectors from the permutation 
to "quantum" basis with the help of (10) leads to

$$
\begin{aligned}
& |\widetilde{\psi}\rangle=\mathrm{T}^{-1}|n\rangle=\frac{1}{\sqrt{3}}\left(\begin{array}{c}
n_{1}+n_{2}+n_{3} \\
n_{1}+n_{2} r+n_{3} \mathrm{r}^{2} \\
n_{1} \mathrm{r}+n_{2}+n_{3} \mathrm{r}^{2}
\end{array}\right), \\
& |\widetilde{\phi}\rangle=\mathrm{T}^{-1}|m\rangle=\frac{1}{\sqrt{3}}\left(\begin{array}{c}
m_{1}+m_{2}+m_{3} \\
m_{1}+m_{2} \mathrm{r}+m_{3} \mathrm{r}^{2} \\
m_{1} \mathrm{r}+m_{2}+m_{3} \mathrm{r}^{2}
\end{array}\right) .
\end{aligned}
$$

Projections of the vectors onto two-dimensional invariant subspace are:

$$
|\psi\rangle=\frac{1}{\sqrt{3}}\left(\begin{array}{l}
n_{1}+n_{2} \mathrm{r}+n_{3} \mathrm{r}^{2} \\
n_{1} \mathrm{r}+n_{2}+n_{3} \mathrm{r}^{2}
\end{array}\right), \quad|\phi\rangle=\frac{1}{\sqrt{3}}\left(\begin{array}{l}
m_{1}+m_{2} \mathrm{r}+m_{3} \mathrm{r}^{2} \\
m_{1} \mathrm{r}+m_{2}+m_{3} \mathrm{r}^{2}
\end{array}\right) .
$$

Constituents of Born's probability (3) for the two-dimensional subsystem are

$$
\begin{gathered}
\langle\psi \mid \psi\rangle=\mathrm{Q}_{3}(n, n)-\frac{1}{3} \mathrm{~L}_{3}(n)^{2}, \\
\langle\phi \mid \phi\rangle=\mathrm{Q}_{3}(m, m)-\frac{1}{3} \mathrm{~L}_{3}(m)^{2}, \\
|\langle\phi \mid \psi\rangle|^{2}=\left(\mathrm{Q}_{3}(m, n)-\frac{1}{3} \mathrm{~L}_{3}(m) \mathrm{L}_{3}(n)\right)^{2},
\end{gathered}
$$

where $\mathrm{L}_{\mathrm{N}}(n)=\sum_{i=1}^{\mathrm{N}} n_{i}$ and $\mathrm{Q}_{\mathrm{N}}(m, n)=\sum_{i=1}^{\mathrm{N}} m_{i} n_{i}$ are linear and quadratic permutation invariants, respectively.

Note that:

1. Expressions (11)-(13) are combinations of the invariants of permutation representation.

2. Expressions (11) and (12) are always positive rational numbers for $|n\rangle$ and $|m\rangle$ with different components.

3. Conditions for destructive quantum interference — vanishing Born's probability — are determined by the equation

$$
3\left(m_{1} n_{1}+m_{2} n_{2}+m_{3} n_{3}\right)-\left(m_{1}+m_{2}+m_{3}\right)\left(n_{1}+n_{2}+n_{3}\right)=0 .
$$

This equation has infinitely many solutions in natural numbers. An example of such a solution is: $|n\rangle=\left(\begin{array}{l}1 \\ 1 \\ 2\end{array}\right), \quad|m\rangle=\left(\begin{array}{l}1 \\ 3 \\ 2\end{array}\right)$.

Thus, we have obtained essential features of quantum behavior from "permutation dynamics" and "natural" interpretation (7) of quantum amplitude by a simple transition to invariant subspaces. 
Any permutation representation contains $(\mathrm{N}-1)$-dimensional invariant subspace. The inner product in this subspace can be expressed in terms of the permutation invariants by the formula

$$
\langle\phi \mid \psi\rangle=\mathrm{Q}_{\mathrm{N}}(m, n)-\frac{1}{\mathrm{~N}} \mathrm{~L}_{\mathrm{N}}(m) \mathrm{L}_{\mathrm{N}}(n) .
$$

The identity $\mathrm{Q}_{\mathrm{N}}(n, n)-\frac{1}{\mathrm{~N}} \mathrm{~L}_{\mathrm{N}}(n)^{2} \equiv \frac{1}{\mathrm{~N}^{2}} \sum_{i=1}^{\mathrm{N}}\left(\mathrm{L}_{\mathrm{N}}(n)-\mathrm{N} n_{i}\right)^{2}$ shows explicitly that $\langle\psi \mid \psi\rangle>0$ for $|n\rangle$ with different components $n_{i}$. This inner product does not contain irrationalities for natural $|n\rangle$ and $|m\rangle$.

\section{Icosahedral Group $\mathbf{A}_{5}$}

The icosahedral group $A_{5}$ - the smallest (it consists of 60 elements) simple noncommutative group - plays an important role in mathematics and applications. F. Klein devoted a whole book to it $[8$. In the physical literature the group is often denoted as $\Sigma(60)$. This group has a "physical incarnation": the fullerene $C_{60}$ carbon molecule ("buckyball") has the structure of the Cayley graph of $\mathrm{A}_{5}$ (see Fig. 1). This is clear from the following presentation of $A_{5}$ by generators and relators (products of generators that are equal to the group identity)

$$
\mathrm{A}_{5} \cong\left\langle a, b \mid a^{5}, b^{2},(a b)^{3}\right\rangle .
$$

Let us consider the action of $A_{5}$ on the set $\Omega_{12}$ of icosahedron vertices. This action is transitive but imprimitive, i.e., there is non-trivial partition of $\Omega_{12}$ invariant under the action. This partition — called system of imprimitivity (or block system) - is the following

$$
\left\{\left|B_{1}\right| \cdots\left|B_{i}\right| \cdots\left|B_{6}\right|\right\} \equiv\{|1,7| \cdots|i, i+6| \cdots|6,12|\}
$$

where, the vertex numbering of Fig. 2 is assumed. Each block $B_{i}$ is a pair of two opposite vertices of the icosahedron and $A_{5}$ permutes the blocks amongst themselves as whole entities. We shall denote the correspondence ("complementarity") between opposite vertices by the symbol c ${ }^{\text {c }}$, i.e., if $B_{i}=\{p, q\}$ then $q=p^{\text {c }}$ and $p=q^{\mathrm{c}}$. For the vertex numbering depicted in Fig. 2 the complementarity can be expressed by the formula $p^{\mathrm{c}} \equiv 1+(p+5 \bmod 12)$.

The permutation representation of the action of $A_{5}$ on the vertices of icosahedron has the following decomposition into irreducible components

$$
\overline{\mathbf{1 2}} \cong \mathbf{1} \oplus \mathbf{3} \oplus \mathbf{3}^{\prime} \oplus \mathbf{5} \text { or } \mathrm{T}^{-1}(\overline{\mathbf{1 2}}) \mathrm{T}=\mathbf{1} \oplus \mathbf{3} \oplus \mathbf{3}^{\prime} \oplus \mathbf{5} .
$$

Note, that the group $A_{5}$ has three primitive actions on sets with 5, 6 and 10 elements. The corresponding permutation representations have the following decompositions

$$
\overline{5} \cong 1 \oplus 4, \overline{6} \cong 1 \oplus 5, \overline{10} \cong 1 \oplus 4 \oplus 5 .
$$




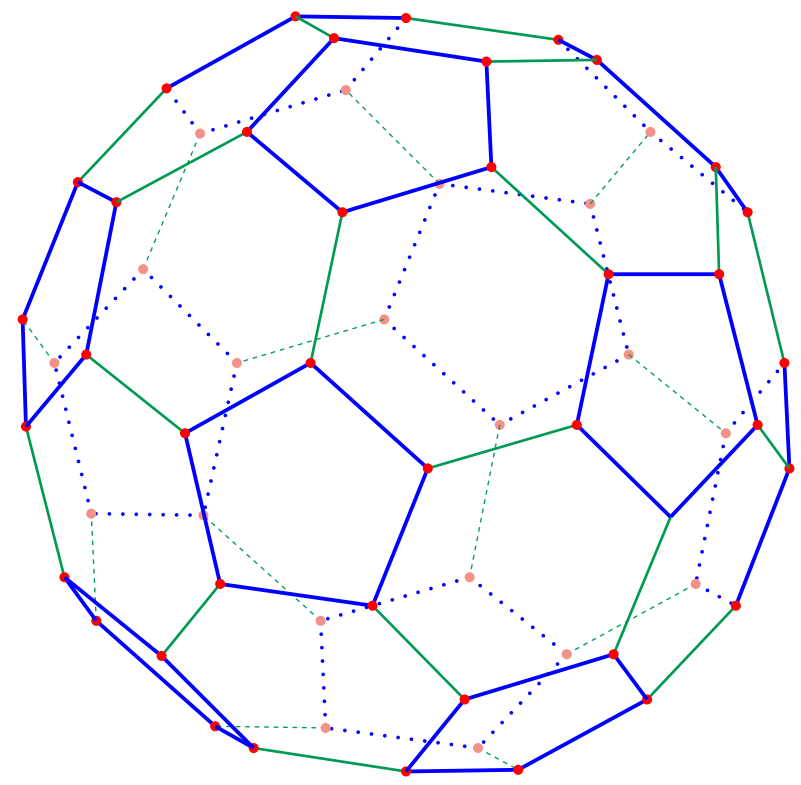

Fig. 1. The Cayley graph of $A_{5}$. The pentagons, hexagons and the links connecting adjacent pentagons correspond to the relators $a^{5},(a b)^{3}$ and $b^{2}$ in presentation (14), respectively.

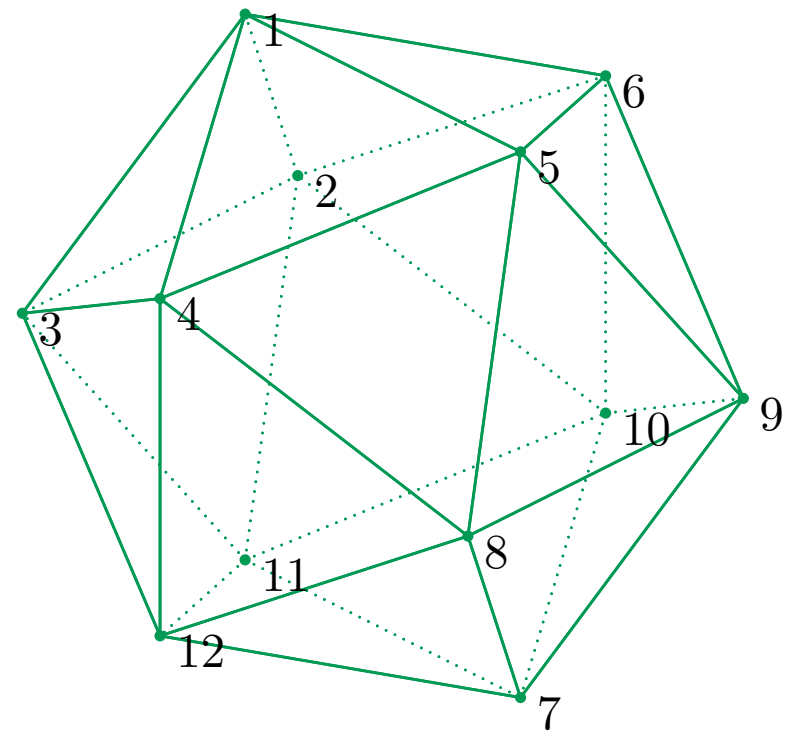

Fig. 2. The icosahedron. Pairs of opposite vertices form the system of imprimitivity. 
With the notations

$\phi=\frac{1+\sqrt{5}}{2}$ the "golden ratio", $\quad \alpha=\frac{\phi}{4} \sqrt{10-2 \sqrt{5}}$,

$\beta=\frac{\sqrt{5} \sqrt{10-2 \sqrt{5}}}{20}, \gamma=\frac{\sqrt{3}}{8}\left(1-\frac{\sqrt{5}}{3}\right), \quad \delta=-\frac{\sqrt{3}}{8}\left(1+\frac{\sqrt{5}}{3}\right)$

a particular form of unitary transformation matrix $\mathrm{T}$ from (15) can be written as

$$
\mathrm{T}=\left(\begin{array}{cccccccccccc}
\frac{\sqrt{3}}{6} & \alpha & \beta & 0 & \alpha & \beta & 0 & \frac{1}{4} & -\frac{1}{2} & 0 & 0 & \frac{\sqrt{15}}{12} \\
\frac{\sqrt{3}}{6} & 0 & \alpha & \beta & -\beta & 0 & \alpha & -\frac{\phi}{4} & 0 & -\frac{1}{2} & 0 & \gamma \\
\frac{\sqrt{3}}{6} & \beta & 0 & \alpha & 0 & -\alpha & -\beta & \frac{\phi-1}{4} & 0 & 0 & -\frac{1}{2} & \delta \\
\frac{\sqrt{3}}{6} & 0 & \alpha & -\beta & -\beta & 0 & -\alpha & -\frac{\phi}{4} & 0 & \frac{1}{2} & 0 & \gamma \\
\frac{\sqrt{3}}{6}-\beta & 0 & \alpha & 0 & \alpha & -\beta & \frac{\phi-1}{4} & 0 & 0 & \frac{1}{2} & \delta \\
\frac{\sqrt{3}}{6} & \alpha & -\beta & 0 & -\alpha & \beta & 0 & \frac{1}{4} & \frac{1}{2} & 0 & 0 & \frac{\sqrt{15}}{12} \\
\frac{\sqrt{3}}{6} & 0 & -\alpha & \beta & \beta & 0 & \alpha & -\frac{\phi}{4} & 0 & \frac{1}{2} & 0 & \gamma \\
\frac{\sqrt{3}}{6} & \beta & 0 & -\alpha & 0 & -\alpha & \beta & \frac{\phi-1}{4} & 0 & 0 & \frac{1}{2} & \delta \\
\frac{\sqrt{3}}{6}-\alpha & \beta & 0 & \alpha & -\beta & 0 & \frac{1}{4} & \frac{1}{2} & 0 & 0 & \frac{\sqrt{15}}{12} \\
\frac{\sqrt{3}}{6}-\alpha & -\beta & 0 & -\alpha & -\beta & 0 & \frac{1}{4} & -\frac{1}{2} & 0 & 0 & \frac{\sqrt{15}}{12} \\
\frac{\sqrt{3}}{6} & 0 & -\alpha & -\beta & \beta & 0 & -\alpha & -\frac{\phi}{4} & 0 & -\frac{1}{2} & 0 & \gamma \\
\frac{\sqrt{3}}{6}-\beta & 0 & -\alpha & 0 & \alpha & \beta & \frac{\phi-1}{4} & 0 & 0 & -\frac{1}{2} & \delta
\end{array}\right) .
$$

Note that the standard computer algebra systems like Maple or Mathematica can not handle such matrices since they can not simplify complicated expressions with irrationalities (especially with nested roots) properly. But if one rewrites the matrix entries as elements of suitable abelian number field $\mathcal{F}$, then the problem of simplification is reduced to a simple one-variable polynomial algebra modulo corresponding cyclotomic polynomial.

The inner products in the invariant subspaces can be expressed in terms of permutation invariants as follows:

$$
\begin{aligned}
\left\langle\Phi_{\mathbf{1}} \mid \Psi_{\mathbf{1}}\right\rangle & =\frac{1}{12} \mathrm{~L}_{12}(m) \mathrm{L}_{12}(n) \\
\left\langle\Phi_{\mathbf{3}} \mid \Psi_{\mathbf{3}}\right\rangle & =\frac{1}{20}\left(5 \mathrm{Q}_{12}(m, n)-5 \mathrm{~A}(m, n)+\sqrt{5}(\mathrm{~B}(m, n)-\mathrm{C}(m, n))\right) \\
\left\langle\Phi_{\mathbf{3}^{\prime}} \mid \Psi_{\mathbf{3}^{\prime}}\right\rangle & =\frac{1}{20}\left(5 \mathrm{Q}_{12}(m, n)-5 \mathrm{~A}(m, n)-\sqrt{5}(\mathrm{~B}(m, n)-\mathrm{C}(m, n))\right) \\
\left\langle\Phi_{\mathbf{5}} \mid \Psi_{\mathbf{5}}\right\rangle & =\frac{1}{12}\left(5 \mathrm{Q}_{12}(m, n)+5 \mathrm{~A}(m, n)-\mathrm{B}(m, n)-\mathrm{C}(m, n)\right)
\end{aligned}
$$


where

$$
\begin{aligned}
& \mathrm{A}(m, n)=\mathrm{A}(n, m)=\sum_{k=1}^{12} m_{k} n_{k^{\mathrm{c}}}, \\
& \mathrm{B}(m, n)=\mathrm{B}(n, m)=\sum_{k=1}^{12} m_{k} \sum_{i \in \mathrm{N}(k)} n_{i}, \\
& \mathrm{C}(m, n)=\mathrm{C}(n, m)=\sum_{k=1}^{12} m_{k} \sum_{i \in \mathrm{N}\left(k^{\mathrm{c}}\right)} n_{i} .
\end{aligned}
$$

In formulas (21) and (22) $\mathrm{N}(k)$ denotes the "neighborhood" of the icosahedron vertex $k$, i. e., the set of vertices adjacent to $k$. For example, $\mathrm{N}(1)=\{2,3,4,5,6\}$ in Fig. 2. Quadratic invariants (20)-(22) are not independent. There is an identity among them:

$$
\mathrm{A}(m, n)+\mathrm{B}(m, n)+\mathrm{C}(m, n)+\mathrm{Q}_{12}(m, n)=\mathrm{L}_{12}(m) \mathrm{L}_{12}(n) .
$$

Inner products (17) and (18) lead to the mentioned above conceptual difficulty with probability when considered separately. Born's probabilities computed separately for the representations $\mathbf{3}$ and $\mathbf{3}^{\prime}$ contain irrationalities. This contradicts the frequency interpretation of probability for finite sets. Of course, this is a consequence of the imprimitivity: one can not move a vertex of the icosahedron without simultaneous moving of its complement in the block. To resolve the contradiction we should consider the complementary representations $\mathbf{3}$ and $\mathbf{3}^{\prime}$ together. The inner product for the representation $\mathbf{3} \oplus \mathbf{3}^{\prime}$ takes the form

$$
\left\langle\Phi_{\mathbf{3} \oplus \mathbf{3}^{\prime}} \mid \Psi_{\mathbf{3} \oplus \mathbf{3}^{\prime}}\right\rangle=\frac{1}{2}\left(\mathrm{Q}_{12}(m, n)-\mathrm{A}(m, n)\right) .
$$

This inner product always gives rational Born's probabilities for vectors with natural "population numbers".

\section{Conclusions}

Let us summarize the main ideas of the paper

1. Quantum mechanics is, in fact, an a priori mathematical scheme based on the fundamental impossibility to trace identity of indistinguishable objects in their evolution — some kind of "calculus of indistinguishables".

2. Any quantum mechanical problem can be reduced to permutations.

3. Quantum interferences are appearances observable in the invariant subspaces of permutation representations - they can be expressed in terms of the permutation invariants.

4. Natural interpretation of quantum amplitudes ( "waves") as vectors of "population numbers" of underlying entities ("particles") leads to the rational quantum probabilities - in accordance with the frequency interpretation of probability for finite sets. 
The idea of natural quantum amplitudes requires verification. If it is valid, then quantum phenomena in different invariant subspaces reveal different manifestations - visible in different "observational settings" - of a single process of permutations of underlying things. One has to interpret the data corresponding to different invariant subspaces. For example, the trivial one-dimensional subrepresentation contained in any permutation representation can be interpreted as the "counter of particles": the permutation invariant $\mathrm{L}_{\mathrm{N}}(n)$ corresponding to this subrepresentation is the total number of particles. Interpretation of data in other invariant subspaces requires careful study.

Acknowledgment. The author is grateful to Yuri Blinkov and Vladimir Gerdt for fruitful discussions and for their help in preparing the paper. The work was partially supported by the grants 01-01-00200 from the Russian Foundation for Basic Research and 3810.2010.2 from the Ministry of Education and Science of the Russian Federation.

\section{References}

1. Altarelli, G., Feruglio, F.: Discrete flavor symmetries and models of neutrino mixing. Rev. Mod. Phys. 82(3) 2701-2729 (2010)

2. Blum, A., Hagedorn, C.: The Cabibbo Angle in a Supersymmetric D14 Model. Nucl. Phys. B821, 327-353 (2009)

3. GAP - Groups, Algorithms, Programming - a System for Computational Discrete Algebra. http://www.gap-system.org/

4. Hall M., Jr.: The Theory of Groups. Macmillan, New York (1959)

5. Harrison, P.F., Perkins, D.H., Scott W.G.: Tri-bimaximal mixing and the neutrino oscillation data. Phys. Lett. B 530, 167 (2002); arXiv: hep-ph/0202074

6. Harrison, P.F., Scott, W.G.: Permutation symmetry, Tri-bimaximal neutrino mixing and the S3 group characters. Phys. Lett. B 557, 76 (2003); arXiv: hep-ph/0302025

7. Ishimori, H., Kobayashi, T., Ohki, H., Okada, H., Shimizu, Y., Tanimoto, M.: Non-abelian discrete symmetries in particle physics. Prog. Theor. Phys. Suppl. 183, 1-173 (2010); arXiv:1003.3552

8. Klein, F. Vorlesungen über das Ikosaeder. Leipzig: Teubner, 1884.

9. Kornyak, V.V.: Quantization in discrete dynamical systems. J. Math. Sci. 168(3), 390-397 (2010)

10. Kornyak, V.V.: Structural and Symmetry Analysis of Discrete Dynamical Systems. In: Cellular Automata. Nova Science Publishers, Inc., New York, pp. 119 163 (2011); http://arxiv.org/abs/1006.1754

11. Ludl, P.O.: Systematic Analysis of Finite Family Symmetry Groups and Their Application to the Lepton Sector, arXiv:0907.5587

12. McKay, B.D. (1981). Practical Graph Isomporphism. Congressus Numerantium 30, 45-87, http://cs.anu.edu.au/ bdm/nauty/PGI

13. Nakamura, K. et al. (Particle Data Group): The review of particle physics. J. Phys. G 37, 075021, 1-1422 (2010)

14. Shafarevich, I. R. Basic Notions of Algebra. Berlin, New York: Springer, 1990.

15. Smirnov, A. Yu.: Discrete Symmetries and Models of Flavor Mixing. arXiv:1103.3461 (2011) 14 p. 